\title{
Immune subgroups and the tumor microenvironment as a potential new biomarker to assess response to immunotherapy
}

\author{
Shawn Kothari ${ }^{1}$, Everett E. Vokes ${ }^{2,3}$ \\ ${ }^{1}$ Division of Hematology-Oncology, University of Pennsylvania, Philadelphia, PA, USA; ${ }^{2}$ Biological Sciences Division, University of Chicago \\ Medicine, Chicago, IL, USA; ${ }^{3}$ Department of Medicine, University of Chicago Comprehensive Cancer Center, Chicago, IL, USA \\ Correspondence to: Everett E. Vokes. 5841 S. Maryland Avenue, MC 6092, Chicago, IL 60637, USA. Email: evokes@medicine.bsd.uchicago.edu. \\ Provenance: This is an invited article commissioned by the Academic Editor Shi-Tong Yu (Department of General Surgery, Nanfang Hospital, \\ Southern Medical University, Guangzhou, China). \\ Comment on: Chen YP, Wang YQ, Lv JW, et al. Identification and validation of novel microenvironment-based immune molecular subgroups of head \\ and neck squamous cell carcinoma: implications for immunotherapy. Ann Oncol 2019;30:68-75.
}

Submitted Aug 09, 2019. Accepted for publication Aug 19, 2019.

doi: $10.21037 /$ cco.2019.08.15

View this article at: http://dx.doi.org/10.21037/cco.2019.08.15

Immunotherapy has emerged as a new standard of care therapeutic option for patients with recurrent or metastatic head and neck squamous cell carcinoma. Following the recent presentation of Keynote-048, the use of Pembrolizumab either as a single agent or in combination with chemotherapy can be considered as first-line treatment for patients with measurable PD-L1 in their tumor or microenvironment (1). However, therapeutic responses have been variable with many patients achieving only marginal or no clinical benefit. Although PD-L1 expression, tumor-mutation burden (TMB) and other factors are under evaluation, no reliable biomarker has been identified to reliably predict outcomes and determine which patients may benefit the most from therapy. Recently, Chen et al. sought to identify patient subgroups based on immunologic assessment of head and neck tumor microenvironment to help prognosticate immunotherapeutic response as published earlier this year in the Annals of Oncology ("Identification and Validation of Novel Microenvironment-based Immune Molecular Subgroups of Head and Neck Squamous Cell Carcinoma: Implications for Immunotherapy") (2).

Non-negative matrix factorization (NMF) is a biostatistics approach that can be applied to immunology to help identify immune related gene expression profiles from bulk gene expression data generated from biopsy samples. Using a NMF approach, the authors assessed 522 patient samples and found that $40 \%$ of the patients $(211 / 522)$ had high levels of gene expression representing active immune pathways. They labelled this group the Immune Class. Given that immune activity can be either stimulatory or inhibitory, the authors further sub-classified the Immune Class into Active ( $\mathrm{n}=56)$ and Exhausted $(\mathrm{n}=155)$ immune classes based respectively on pro- and anti-inflammatory signals.

The authors then explored the association of these immune subclasses with clinical and pathologic variables. The active immune class was associated with early pathologic T-status, primary site of disease in the oropharynx, HPV positive disease, significantly better overall survival, and improved disease-free survival as compared to the exhausted immune class and the nonimmune class. These results were validated in assessment of six additional publicly available genomic datasets comprising 838 patient samples. In the six validation groups, the immune molecular subgroups were identified and similarly correlated with improved outcomes.

Chen et al.'s work is interesting in that it reminds us how heterogeneous HNSCC can be and encourages further investigation of this NMF classification as a potential new biomarker clinician can assess to determine immunotherapy responsiveness. Given the current absence of a consistently reliable biomarker, additional work in this area is clearly warranted (1,3). At this point, PD-L1 expression remains the most frequently used biomarker but its predictive potential is limited and response rates are variable. As such, researchers have sought to identify additional 
biomarkers, and the assessment of the tumor immune microenvironment has become one potential option. Prior studies have suggested that inflamed environments lead to improved clinical outcomes and the current study appears to corroborate this finding.

Additional work needs to be done to validate this finding, and a prospective trial should be considered with assessment of the tumor microenvironment prior to immunotherapy receipt. In the meantime, we congratulate the authors of this study for their significant contribution to the field of immune-oncology in head and neck cancer.

\section{Acknowledgments}

None.

\section{Footnote}

Conflicts of Interest: The authors have no conflicts of interest to declare.

Cite this article as: Kothari S, Vokes EE. Immune subgroups and the tumor microenvironment as a potential new biomarker to assess response to immunotherapy. Chin Clin Oncol 2019;8(Suppl 1):S24. doi: 10.21037/cco.2019.08.15
Ethical Statement: The authors are accountable for all aspects of the work in ensuring that questions related to the accuracy or integrity of any part of the work are appropriately investigated and resolved.

\section{References}

1. Rischin D, Harrington K, Greil R, et al. Protocolspecified final analysis of the phase 3 KEYNOTE-048 trial of pembrolizumab (pembro) as first-line therapy for recurrent/metastatic head and neck squamous cell carcinoma (R/M HNSCC). J Clin Oncol 2019;37:6000.

2. Chen YP, Wang YQ, Lv JW, et al. Identification and validation of novel microenvironment-based immune molecular subgroups of head and neck squamous cell carcinoma: implications for immunotherapy. Ann Oncol 2019;30:68-75.

3. Ferris RL, Blumenschein G Jr, Fayette J, et al. Nivolumab for Recurrent Squamous-Cell Carcinoma of the Head and Neck. N Engl J Med 2016;375:1856-67. 